THE ASTRONOMICAL JouRNAL, 117:1332-1340, 1999 March

(C) 1999. The American Astronomical Society. All rights reserved. Printed in U.S.A.

\title{
DOUBLE-MODE RR LYRAE VARIABLES IN THE GLOBULAR CLUSTER M3
}

\author{
T. Michael CORWIN ${ }^{1}$ \\ Department of Physics, University of North Carolina at Charlotte, Charlotte, NC 28223; mcorwin@uncc.edu \\ BRUCE W. CARNEY ${ }^{1}$ \\ Department of Physics and Astronomy, University of North Carolina, Chapel Hill, NC 27599; bruce@sloth.astro.unc.edu \\ AND \\ DAVID M. ALLeN \\ Department of Physics, University of North Carolina at Charlotte, Charlotte, NC 28223 \\ Received 1998 September 28; accepted 1998 November 24
}

\begin{abstract}
We present new $B$-band CCD photometry for five double-mode RR Lyrae (RRd) variables (V68, V79, V87, V99, and V166) in the globular cluster M3. The pulsational behavior of the RRd variables is described. V68 and V87 have been known as RRd variables since 1982, V79 was recently discovered as an RRd (Clement et al.), and our data have identified V99 and V166 as RRd variables (Corwin et al.). Earlier studies of V79 and V166 do not show double-mode behavior, which indicates that these stars have only recently become RRd stars. V166 changed its dominant pulsation mode from fundamental to first overtone in the interval 1992 to 1993. The candidate double-mode variables V28 and V126 do not exhibit clear RRd behavior in the 1992-1993 data.
\end{abstract}

Key words: globular clusters: individual (M3) — stars: evolution — RR Lyrae variable

\section{INTRODUCTION}

Sandage, Katem, \& Sandage (1981) suggested that certain variables in the globular cluster M15 with unusually large scatter in their light curves might be pulsating simultaneously in the first-overtone and fundamental radial modes. Cox, Hudson, \& Clancy (1983) and Nemec (1985) confirmed the existence of the M15 double-mode pulsators, and Nemec designated these stars RRd variables. Double-mode variables are particularly useful in that they allow the determination of pulsational masses independently of stellar evolution theory (Cox et al. 1983). Other double-mode pulsators have now been discovered in several globular clusters, including M3. In addition to M15 and M3, other globular clusters with known double-mode pulsators are M68, IC 4499, NGC 2419, and NGC 6426. A list of RRd stars within the globular clusters is given by Clement, Ferance, \& Simon (1993). Since 1993 some additional RRd stars have been discovered in M68 (Walker 1994) and in IC 4499 (Walker \& Nemec 1996) and, of course, M3.

In M3, V68 and V87 were first identified as RRd variables (Goranskij 1981 and H. E. Jorgensen, private communication to A. N. Cox cited in Cox et al. 1983), V79 was added in 1997 (Clement et al. 1997), and V99 and V166 last year (Corwin, Allen, \& Carney 1998). In this paper, we use $B$-band data to determine the first-overtone and fundamental pulsation periods and amplitudes of the five RRd variables.

The existence of double-mode pulsators suggests the possibility that these stars might be switching pulsation modes from overtone to fundamental or vice versa. Cox et al. (1983) pointed out, however, that the theoretical switching time is only about $150 \mathrm{yr}$, much too short to account for the observed number of RRd variables. More precise location of RRd stars on the color-magnitude diagram should

\footnotetext{
${ }^{1}$ Visiting Astronomer, Kitt Peak National Observatory, National Optical Astronomy Observatories, which are operated by AURA, Inc., under cooperative agreement with the National Science Foundation.
}

help address this issue. In any case, the physical mechanism for long-term maintenance of double-mode pulsation is not understood.

One of the long-standing problems in astronomy is the division of globular clusters into two distinct groups according to the mean values of the periods of the RRab Lyraes (Oosterhoff 1939). The Oosterhoff effect is related to, but not entirely determined by, metallicity. In general, intermediate-poor metallicity clusters are Oosterhoff type I (OoI) clusters, and very poor metallicity clusters are type II (OoII) clusters. M3, with $[\mathrm{Fe} / \mathrm{H}]=-1.4$ (Carretta \& Gratton 1997), is an OoI cluster. (However, some clusters with metallicity near that of M3 are OoII clusters.) Differences in the properties and evolutionary behavior of double-mode RR Lyraes in OoI and OoII clusters may be helpful in understanding the nature of the Oosterhoff dichotomy.

\section{OBSERVATIONS AND REDUCTIONS}

\subsection{Observations}

The CCD images used in this study were obtained with the No. $10.9 \mathrm{~m}$ telescope at the Kitt Peak National Observatory. The field was first observed for six nights, 1992 May $12 / 13-17 / 18$, and follow-up observations were made on seven nights, 1993 April 7/8-13/14. The $2048 \times 2048$ T2KA CCD was used. For the data reported here, the "Harris" $B$ filter was used, with an exposure time of $500 \mathrm{~s}$. The $24 \mu \mathrm{m}$ pixels of the T2KA CCD, and the $\mathrm{f} / 7.5$ focus gave a pixel scale of 0.69 and a field of view of $23^{\prime}$. None of the nights in 1992 were photometric. Four of the seven 1993 nights were photometric. All 13 nights were clear enough for differential photometry.

\subsection{Data Reductions}

The raw data frames were processed following standard procedures to remove the bias, trim the pictures, and divide by mean dome flats obtained using color-balanced filters. Also an illumination correction using twilight flats and the MKSKYCOR command in IRAF was included in the pro- 
cessing. No attempt was made to recover bad pixels or columns. The raw magnitudes were derived using ALLSTAR in DAOPHOTX in IRAF version 2.10.

The night of 1993 April 9/10 was photometric and we observed 23 standard stars selected from Landolt (1983). The range in $(B-V)$ was from -0.162 to +1.738 . After correcting for a shutter delay time of $-0.06 \mathrm{~s}$, the resulting transformation equations were

$$
\begin{gathered}
V-v_{0}=[0.000 \pm 0.007](b-v)_{0}-[4.280 \pm 0.009] \\
(B-V)=[1.001 \pm 0.005](b-v)_{0}-[0.163 \pm 0.008]
\end{gathered}
$$

\subsection{Reference Stars}

Twelve stars in the field were selected as local magnitude standards against which we could perform relative photometry. All were stars on or near the horizontal branch, six on the red side and six on the blue side of the instability strip. The magnitude difference of each individual star against the mean was plotted as a function of time to check for signs of variability. All appear to be photometrically stable.

\section{RESULTS}

We determined the primary periods for the $\mathrm{R} R \mathrm{~d}$ variables with a computer program that utilized Stellingwerf's (1978) phase dispersion minimization (PDM) technique with a $(5,2)$ bin structure. The $\Theta$ statistic of the technique is a measure of the variance of the data with respect to the mean light curve for a particular period. To search for the secondary period, we derived a mean light curve by fitting a cubic spline interpolating function to the bin means and then measured the residuals from this curve and applied the PDM technique to the residuals to determine the secondary period. This technique was then applied to the residuals from the secondary light curve to search for additional periodicity. In all cases the interaction frequency, $1 / P_{1}$ $+1 / P_{0}$, was found and subtracted. No additional interaction or harmonic frequencies produced recognizable light curves. We then corrected the magnitudes for both the primary and secondary pulsations by subtracting the appropriate mean curves from the raw magnitudes. The periods and amplitude ratios for the RRd variables in M3 are given in Table 1. (The amplitude ratios reported in this paper are somewhat different from those reported by us in Corwin et al. 1998. This is primarily because the light curves used for the Information Bulletin of Variable Stars paper did not have the interaction frequency, $1 / P_{1}+1 / P_{0}$, subtracted.)

\subsection{Notes on Individual Stars: New RRd Variables}

V166.-Nemec \& Clement (1989), using data taken between 1920 and 1926, concluded that a definitive period for V166 could not be determined but that 0.32727 fitted the observations better than the published period of 0.485622 (Sawyer-Hogg 1973) or an alias at 0.48572. Szeidl (1973) using data from 1938 to 1962 found strong lightcurve variation and concluded that the period is probably about 0.486 . Neither of these studies saw evidence of a first-overtone period.

Our 1992 data show a minimum in the Stellingwerf's $\Theta$ statistic versus period plot at about 0.326 (Fig. 1a). The light curve for a period of 0.326 has a clear RRab shape and amplitude. The actual period is probably associated with the other obvious $\Theta$ statistic minimum at about 0.485 (for which 0.326 is an alias). The third lowest $\Theta$ statistic minimum is near 0.359 , roughly the expected first-overtone period if V166 is a double-mode pulsator.

The 1992 data were analyzed using the PDM technique described above. The mean curves for the secondary period (in this case the first overtone) and the interaction period were subtracted from the raw data, and night-to-night phase differences in the light curves were used to determine the best period for the primary pulsation. The value that gives the least spread in the light curve is 0.4856 (Fig. 2c). The maxima for each night, however, are clearly out of phase. There appears to be a systematic phase shift from night 1 to night 2 to night 3 . However nights 4 , 5 , and 6 do not continue this pattern. It is as if there is a 0.32 gap in phase between nights 3 and 4, with the phase-shift pattern continuing from there for nights 4,5 , and 6 . This same pattern can also be seen in the raw data (Fig. 2a). The $\Theta$ statistic plot of the residuals for the 1992 data shows a clear minimum at 0.3595 (Fig. $1 b$ ).

The amplitudes for the 1992 data are about 1.0 mag for the fundamental, primary pulsation and $0.30 \mathrm{mag}$ for the first-overtone, secondary pulsation.

The $\Theta$ statistic plot for the 1993 data (taken just 11 months after the 1992 data) clearly shows a change in the dominant mode of oscillation for V166 (Fig. 3a). The $\Theta$ statistic now has a minimum for the first-overtone oscillation with the fundamental oscillation showing a less strong minimum. As with the 1992 data, the first-overtone period (now the primary period) appears to be 0.3595 (Fig. $4 c$ ). The expected period for the secondary, fundamental oscillation (based on $P_{0} / P_{1}=0.746$ ) is about 0.482 . This value gives a better light curve than 0.4856 , the fundamental period from the 1992 data. It is possible that the period of the fundamental did change in connection with the change in dominant pulsation mode. Clement et al. (1997) noted a decrease in fundamental period of 0.0033 for V79 between 1962, when it was not a double-mode pulsator, and

TABLE 1

Properties FOR THE RRd StARS IN M3

\begin{tabular}{cllcccc}
\hline \hline Star & \multicolumn{1}{c}{$P_{1}$} & \multicolumn{1}{c}{$P_{0}$} & $A_{1} / A_{0} 1992$ & $A_{1} / A_{0} 1993$ & $A_{1} / A_{0} 1996^{\mathrm{a}}$ & $A_{1} / A_{0} 1920-26^{\mathrm{a}}$ \\
\hline V68 $\ldots \ldots .$. & 0.356 & 0.479 & 1.1 & 1.1 & 1.12 & 0.72 \\
V79 $\ldots \ldots .$. & 0.3575 & 0.4797 & 1.6 & 1.6 & 1.73 & $<0.2$ \\
V87 $\ldots \ldots$. & 0.358 & 0.480 & 2.6 & 2.6 & $\ldots$ & $\ldots$ \\
V99 $\ldots \ldots .$. & 0.3611 & 0.4835 & 1.9 & 2.2 & $\ldots$ & $\ldots$ \\
V166 ...... & 0.3595 & 0.482 & 0.30 & 1.1 & $\ldots$ & $\ldots$ \\
\hline
\end{tabular}

${ }^{\text {a }}$ From Clement et al. 1997. 


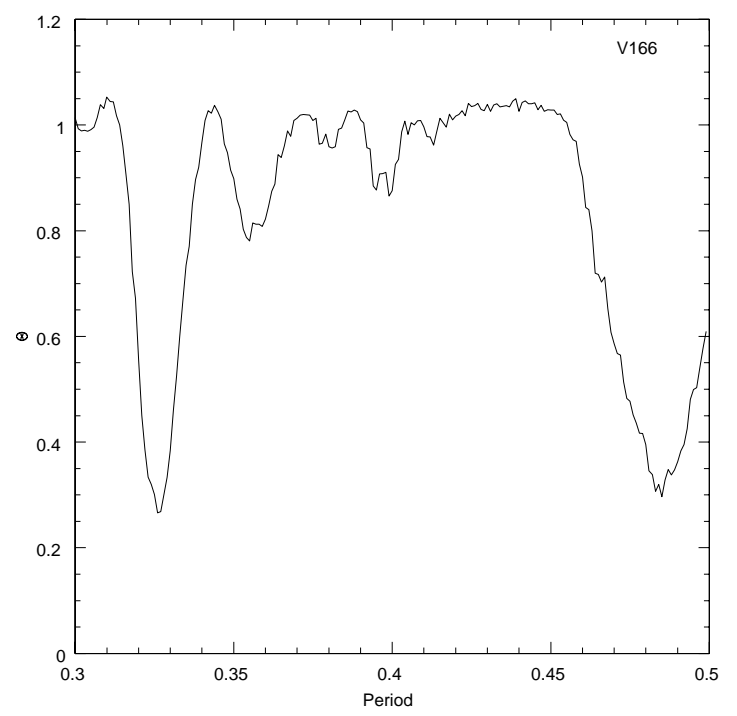

FIG. $1 a$

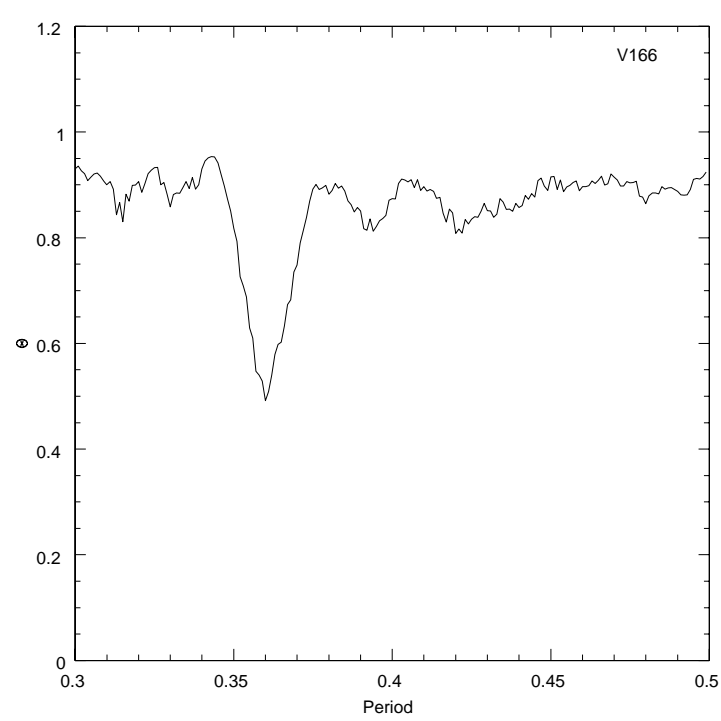

FIG. $1 b$

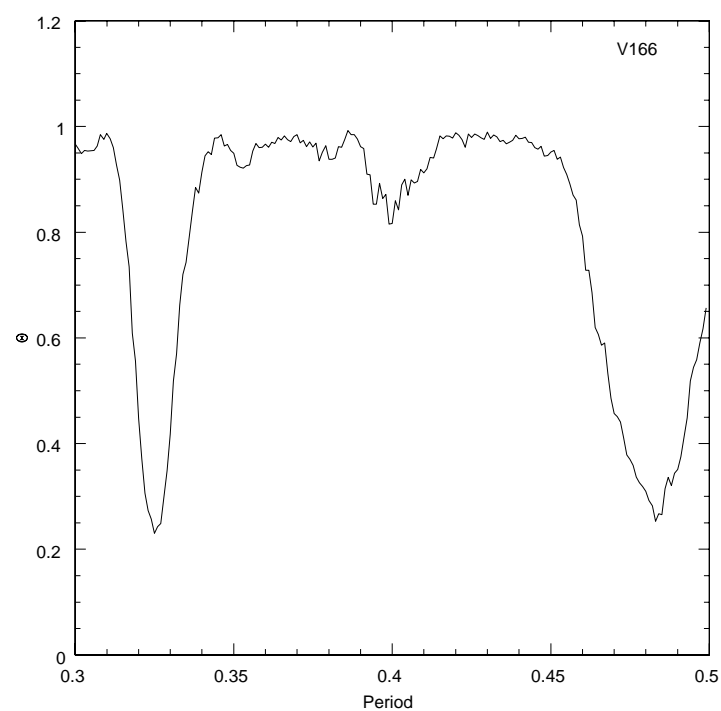

FIG. $1 c$

FIG. 1.-Stellingwerf's $\Theta$ statistic vs. period for V166 1992 data; (a) for the raw data, $(b)$ for the corrected secondary data, and $(c)$ for the corrected primary data.

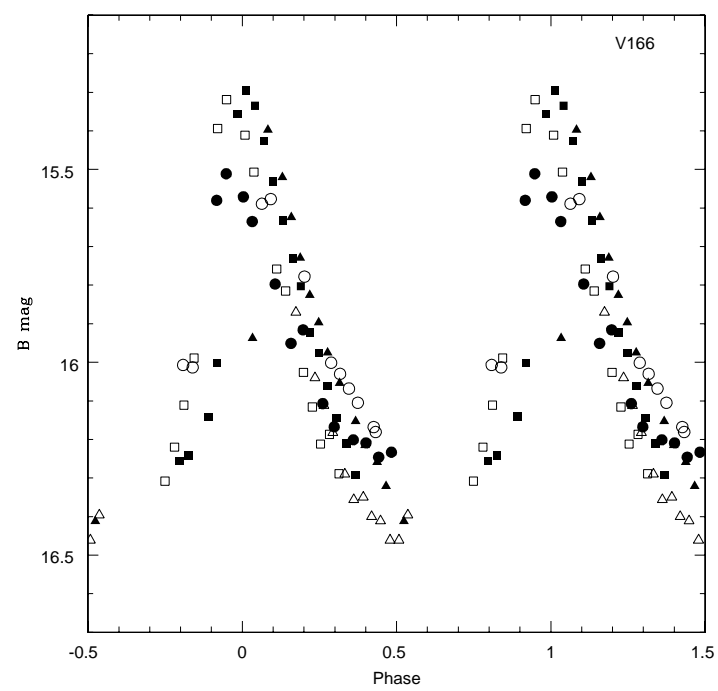

FIG. $2 a$

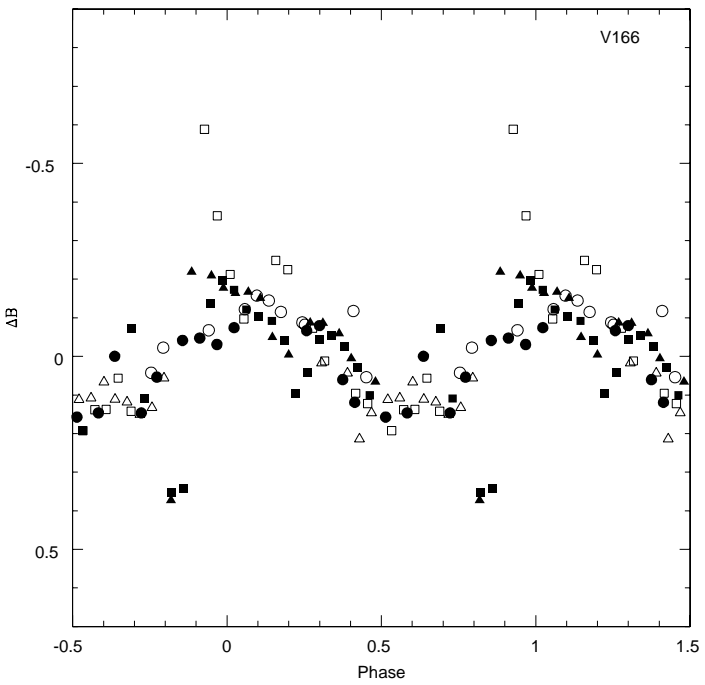

FIG. $2 b$

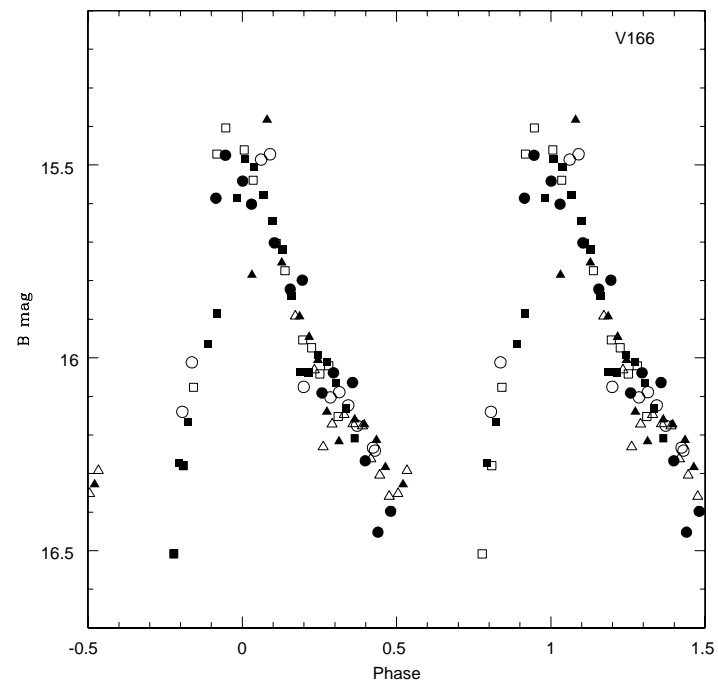

FIG. $2 c$

Fig. 2.- - Light curves for V166 1992 data, night 1 (open squares), night 2 ( filled squares), night 3 (open circles), night 4 (filled circles), night 5 (open triangles), night 6 (filled triangles), (a) for the raw data, $(b)$ for the secondary pulsation, and (c) for the primary pulsation. 


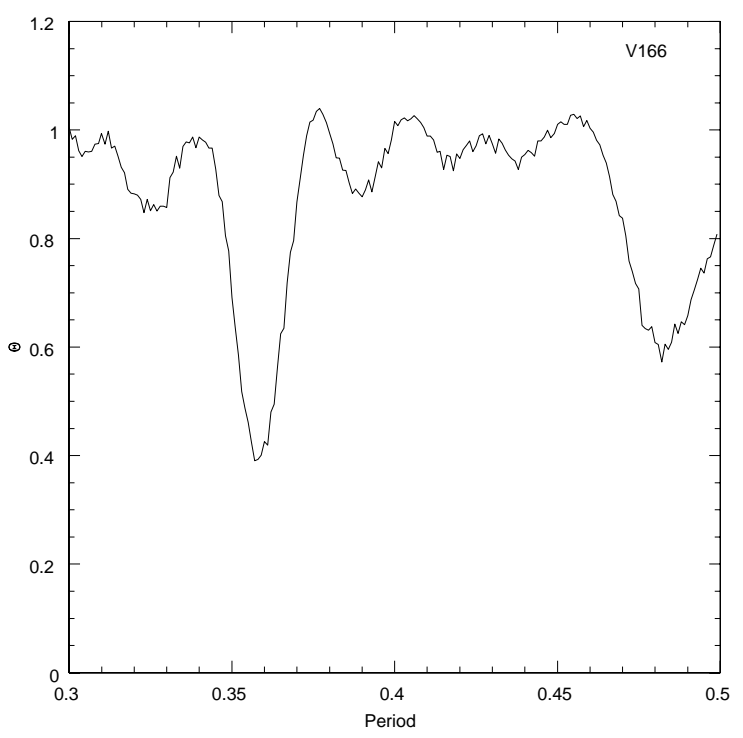

FIG. $3 a$

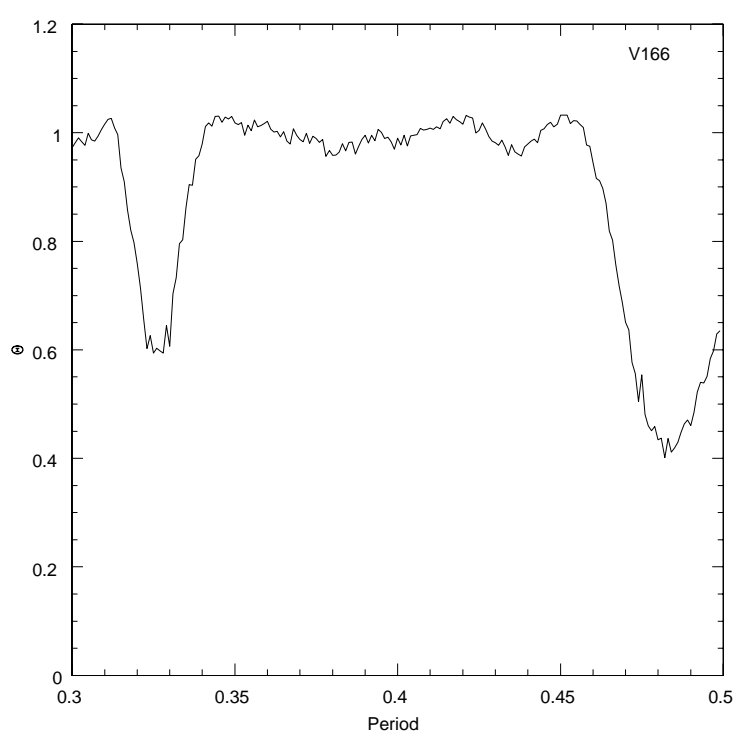

FIG. $3 b$

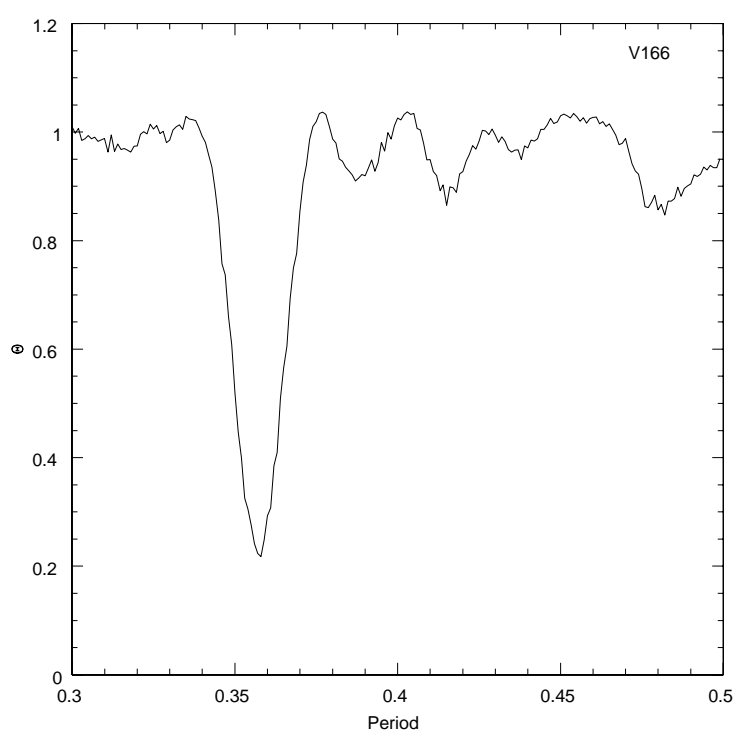

FIG. $3 c$

Fig. 3.-Same as Fig. 1 for V166 1993 data

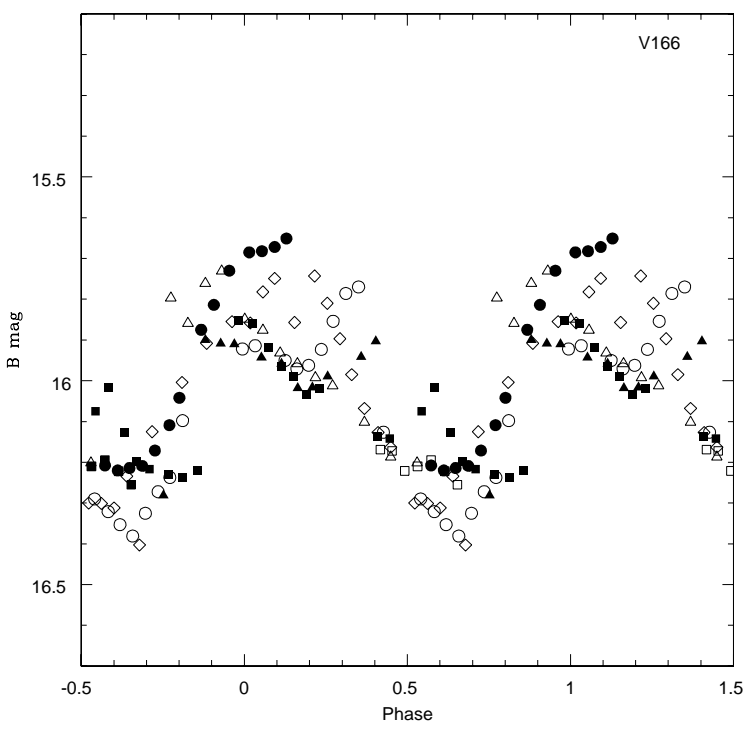

FIG. $4 a$

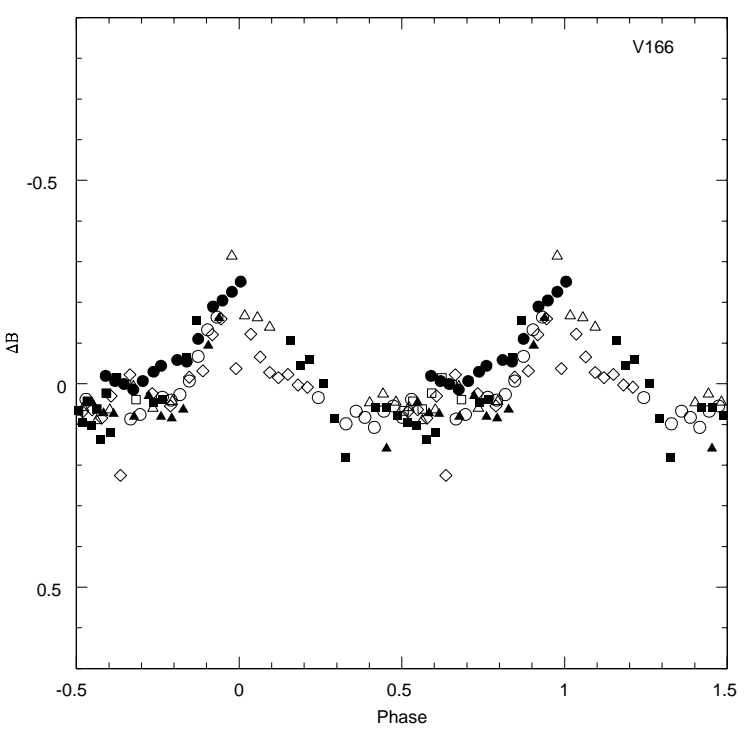

FIG. $4 b$

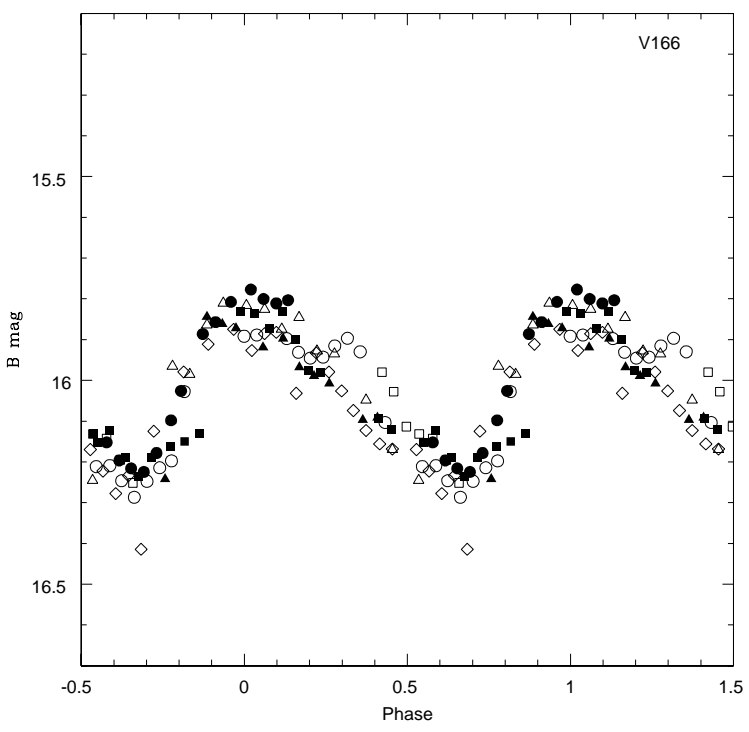

FIG. $4 c$

FIG. 4.-Same as Fig. 2 for V166 1993 data, night 7 (diamonds) 
1996, when it was a double-mode pulsator with a dominant first-overtone pulsation.

The amplitudes for the 1993 data are $0.42 \mathrm{mag}$ for the first-overtone, primary pulsation and 0.39 mag for the fundamental, secondary pulsation.

V99.-The updated compilation of the Sawyer-Hogg Catalog (1973) (C. Clement, private communication) does not list a period for V99.

The PDM technique applied to the 1992 and 1993 data for V99 gives a corrected primary period of 0.3611 , which yields reasonable light curves. Figures 5 and 6 show the light curves for V99. The $\Theta$ statistic plots for the 1992 and 1993 secondary pulsations suggest minima somewhere between 0.48 and 0.49 . However, the minima seem unusually broad and irregular. The minimum for the 1992 data occurs at 0.4835 . Light curves for this period show the secondary, fundamental pulsation to have a small amplitude. The 1993 data, which have a very unusual minimum in the power spectrum, appear to have night-to-night phase shifts. However, there is no period close to 0.48 that will remove the phase shifts.

The amplitude of the first-overtone, primary pulsation may have increased slightly from 1992 to 1993, from about $0.57 \mathrm{mag}$ to about $0.65 \mathrm{mag}$. The amplitude of the fundamental, secondary light curves appears to be around 0.3 mag.

\subsection{Notes on Individual Stars: Previously Known RRd Variables}

V68.-Bailey (1913) identified V68 as an RRab star with a period of about one-half day. Martin (1942) noted that the light curve had the appearance of an irregular RRc variable and that the amplitude was changing rapidly between 0.2 and $1 \mathrm{mag}$. He derived a period of $0 \mathrm{~d} 355974$. Unfortunately, his observational data were never published. Goranskij (1981) recognized V68 as a double-mode pulsator with a dominant first overtone at 0.3559732 and a secondary fundamental at 0.4778805 . Later, Nemec \& Clement (1989), reanalyzing the 1920-1926 data, found V68 to have been an $\mathrm{RRd}$ at that time but with the fundamental mode dominant. At the time no other RRd was known to have ever had a dominant fundamental mode. (In the MACHO Project study of double-mode RR Lyrae stars [Alcock et al. 1997], almost all RRd stars had first-overtone amplitudes greater than the fundamental amplitude.) Nemec \& Clement's reanalysis of Goranskij's data favors a fundamental period of 0.478512 . Nemec \& Clement also found V68 to exhibit Blazhko behavior.

It seems clear that sometime between 1926 and 1981, V68 changed its dominant mode of pulsation from the fundamental mode to the first overtone. V68 was included in the 1996 data of Clement et al. (1997). They found the first overtone to be dominant with an amplitude ratio of first overtone to fundamental of 1.12 .

Our data show V68 as a double-mode pulsator with the first-overtone pulsation being dominant. The $\Theta$ statistic plots give periods that were consistent with those given in Clement et al. (1997), 0.356 for the primary, first-overtone pulsation and 0.479 for the secondary, fundamental pulsation (Fig. 7). The amplitudes are about $0.50 \mathrm{mag}$ for the primary pulsation and about 0.44 mag for the secondary pulsation.

V79.- The updated Sawyer-Hogg Catalog lists V79 as an RRab Blazhko with a period of 0 d 483300 . Nemec \&

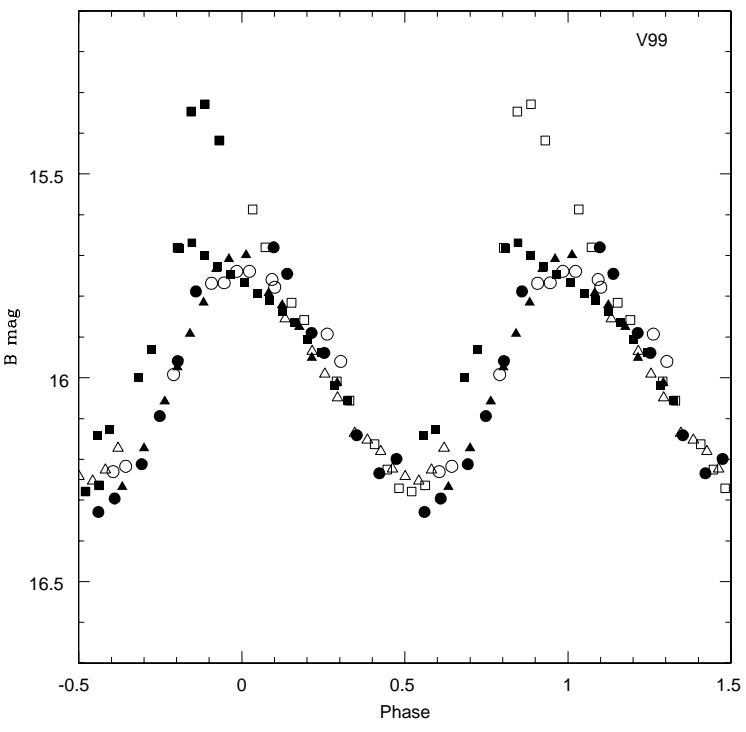

FIG. $5 a$

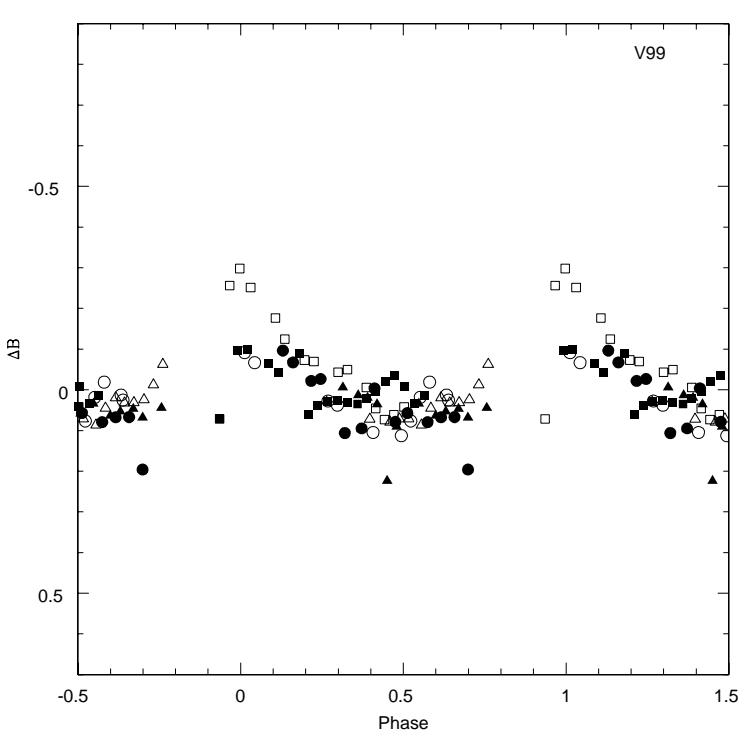

FIG. $5 b$

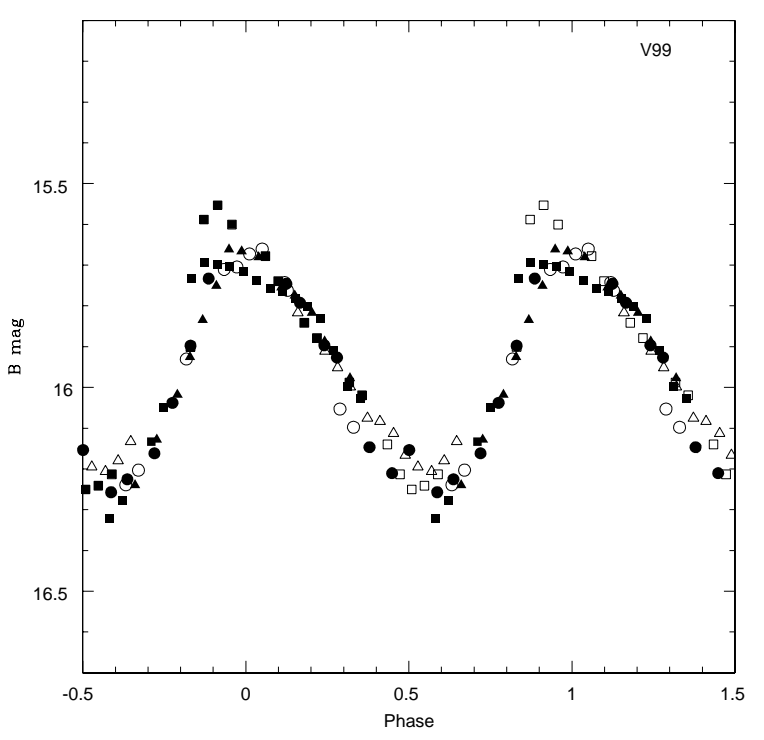

FIG. $5 c$

Fig. 5.-Same as Fig. 2 for V99 1992 data 


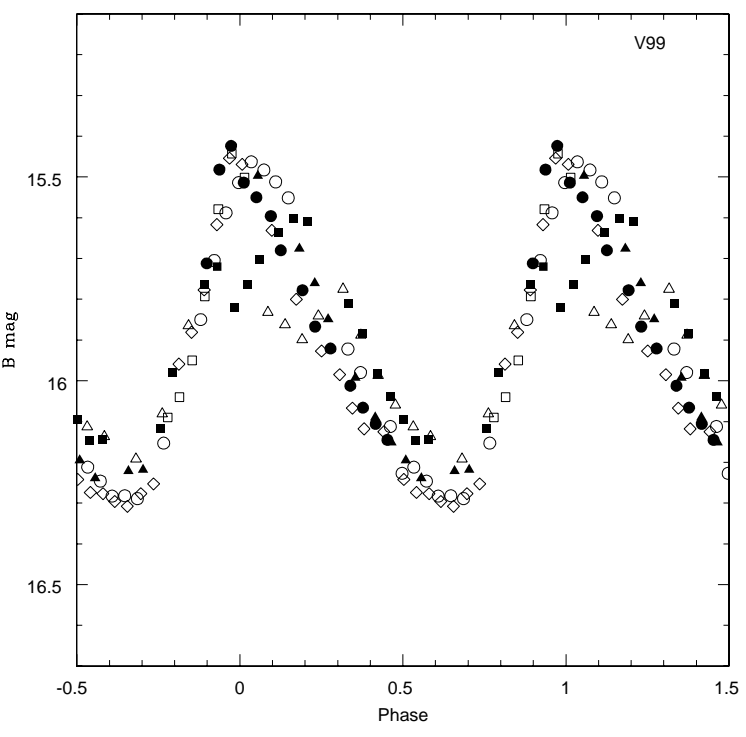

FIG. $6 a$

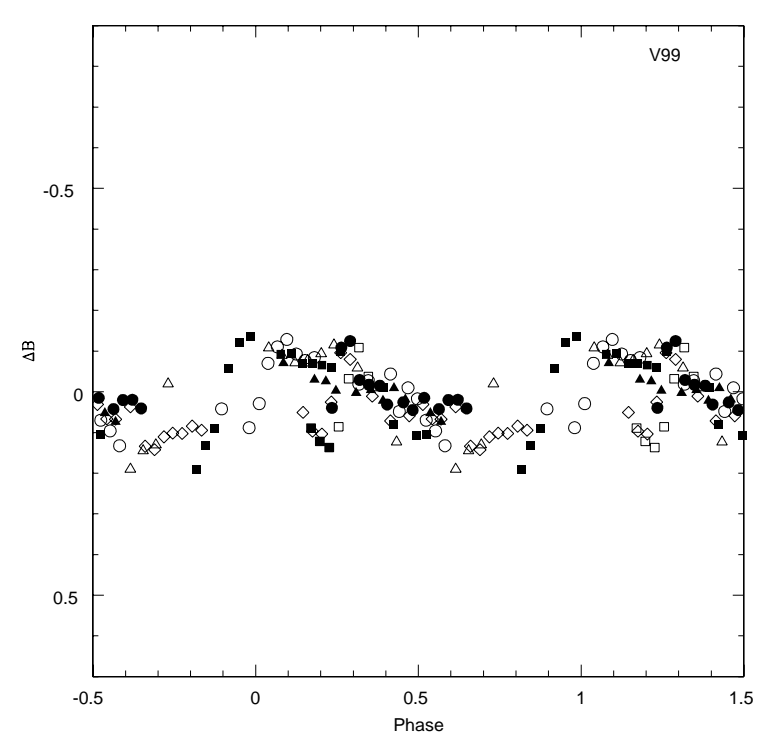

FIG. $6 b$

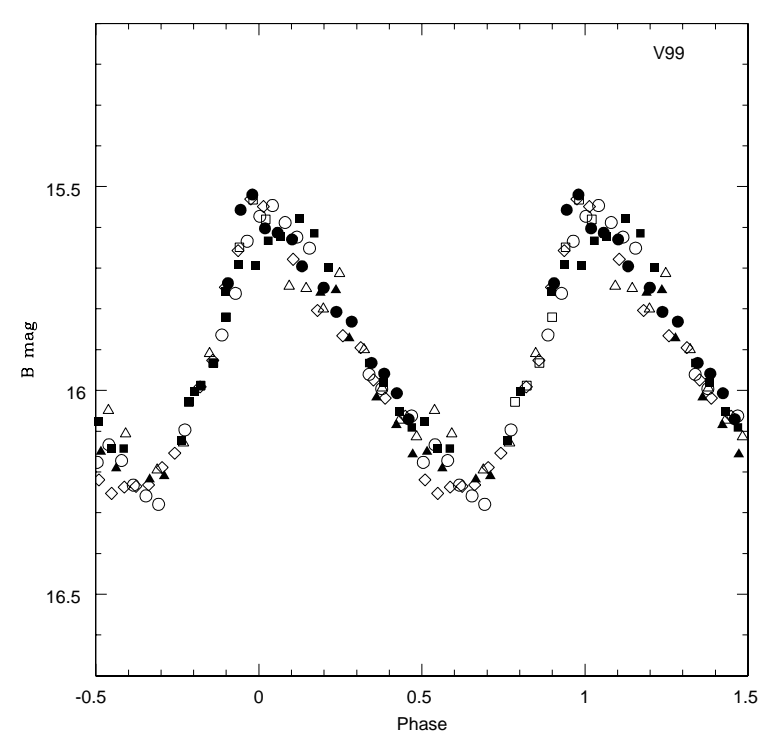

FIG. $6 c$

FIG. 6.- Same as Fig. 4 for V99 1993 data
Clement (1989), using data taken between 1920 and 1926, showed V79 to have night-to-night scatter but were unable to detect a first-overtone component to the light curve. They concluded that if there were any first-overtone oscillations, they would have to have an amplitude less than 0.25 mag. Clement et al. (1997) analyzed data in the interval 1938 to 1962 , and again no first-overtone oscillation was detected, though there were variations in the amplitude. Using data from 1996, however, they found that V79 was an RRd variable with a dominant first-overtone oscillation at 0.358 . They also found that the period of the fundamental oscillation had decreased substantially in the $35 \mathrm{yr}$ interval, from 0.4833 to 0.480 .

Our data also show V79 as an RRd variable with primary, first-overtone period of 0.3575 and a secondary, fundamental period of 0.4797 (Fig. 8). The amplitude of the primary is about $0.40 \mathrm{mag}$, and the secondary is about 0.25 mag.

V87.-The double-mode oscillation of V87 was recognized in 1982 (H. E. Jorgensen, private communication to A. N. Cox, cited in Cox et al. 1983). Jorgensen reported a dominant first overtone with period 0.3574814 and a fundamental with period 0.4797 . Nemec \& Clement's reanalysis for earlier data shows clear RRd behavior in the 1920-1926 data.

Our data are consistent with these periods (Fig. 9). The amplitude of the primary pulsation is about $0.51 \mathrm{mag}$, and the secondary is about $0.20 \mathrm{mag}$.

\subsection{Notes on Individual Stars: Suspected RRd Variables}

V28.-Nemec \& Clement (1989) cite V28 as a candidate double-mode star. In the 1920-1926 data they analyzed, it shows an extremely irregular light curve, and its period of about $0 \mathrm{~d} 47$ day is what would be expected for the fundamental mode of an M3 RRd star. They were unable to find evidence for a secondary period and concluded that V28 was probably a Blazhko variable. The light curve for V28 was much smoother in our data (Fig. 10), and we were also unable to find evidence for a secondary period. Our data do show a slightly different light curve in 1993 than in 1992 with a clear decrease of about 0.2 mag in amplitude. Our best period for the data was $0 \mathrm{~d} 468600$, somewhat less than the Nemec \& Clement period of 0.470120 .

V126. - Nemec \& Clement (1989) also cite V126 as a candidate double-mode star. In the 1920-1926 data they analyzed, it shows possible irregularity in the light curve, and its period of about 0.35 is what would be expected for the first-overtone mode of an M3 RRd star. As with V28, they did not find clear evidence for a secondary period. The period for V126 is $0 \mathrm{~d} 348405$, and it has a fairly regular light curve in our data (Fig. 11). The $\Theta$ statistic plot showed a hint of a secondary period at about $0 \mathrm{~d} 48$, but with the very small residual magnitudes, no light curve could be produced. There does seem to be a possible increase in amplitude of about $0.1 \mathrm{mag}$ from 1992 to 1993.

\section{DISCUSSION}

The periods of RR Lyrae variables can be established to a very high level of accuracy, and, thus, small changes in the pulsation period can easily be determined. Changes in the period are related to changes in the structure of the star, and thus to evolutionary effects, through the pulsation equation. Attempts to use period changes in RR Lyraes to determine horizontal-branch evolution have been frus- 


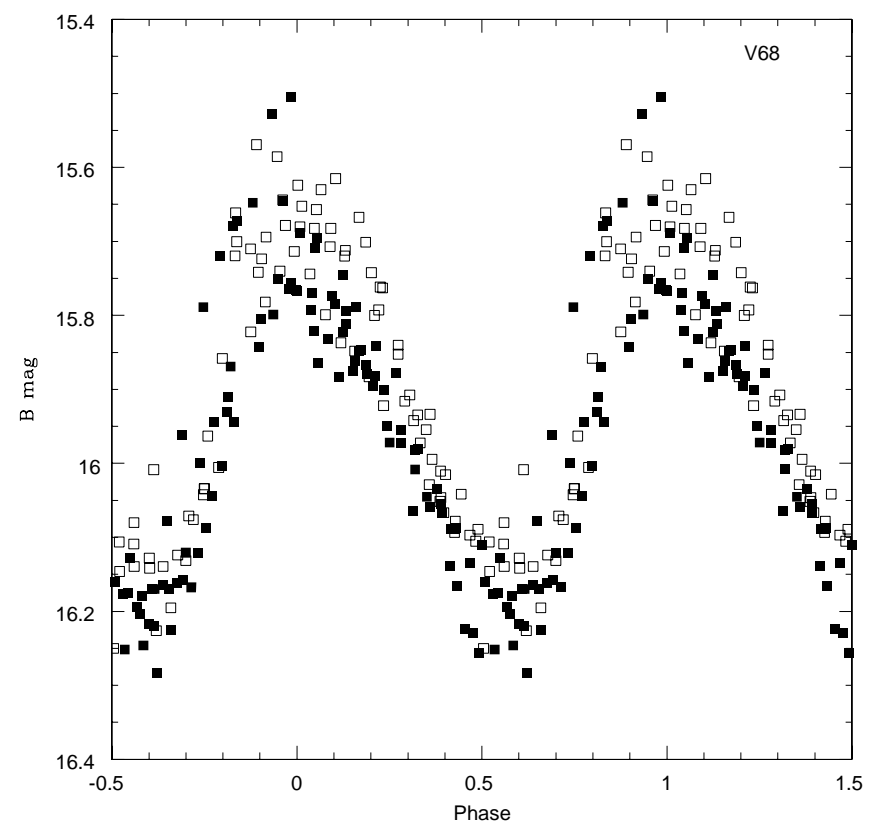

FIG. $7 a$

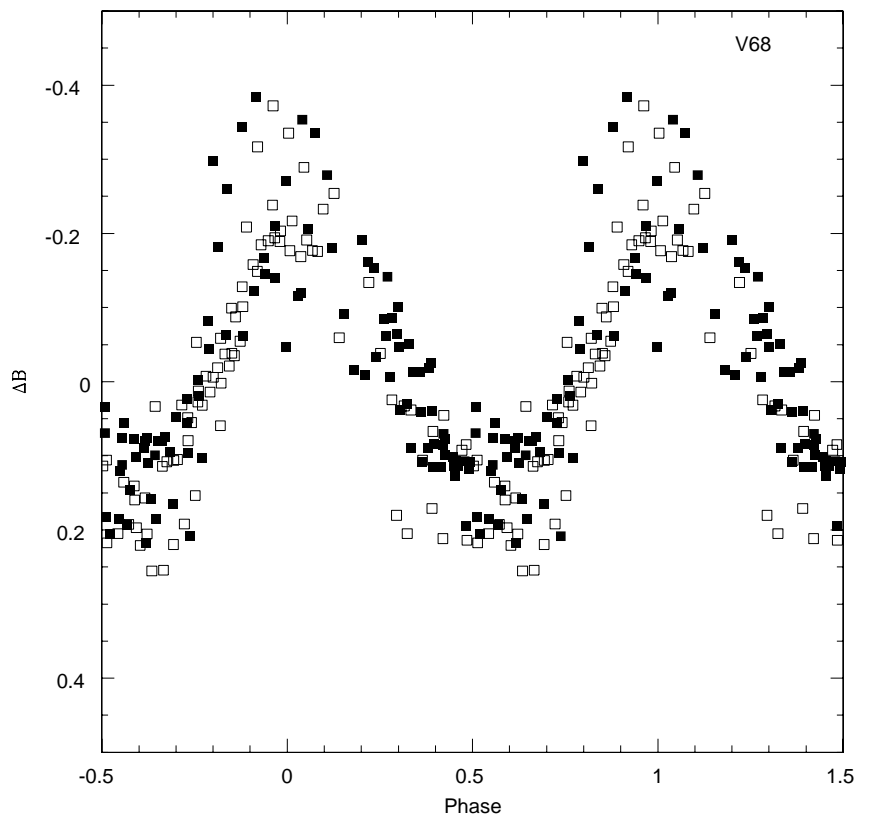

FIG. $7 b$

FIG. 7.-Light curves for V68 1992 (open squares) and 1993 ( filled squares) data, (a) primary pulsation, and (b) secondary pulsation

trated, however, by the presence of "noise" in the period changes. Iben \& Rood (1970) showed that the observed period changes were much larger than the evolutionary changes predicted by the standard models. However, they argued that the evolutionary changes could be seen in the mean of data dominated by noise.

Smith (1995) presents a summary of recent studies of RR Lyrae period changes. He points out that OoI clusters tend to have small median period changes and roughly equal numbers of stars with increasing and decreasing periods. In OoII clusters, more stars may be increasing in period than decreasing. It has been suggested that the excess of increas-

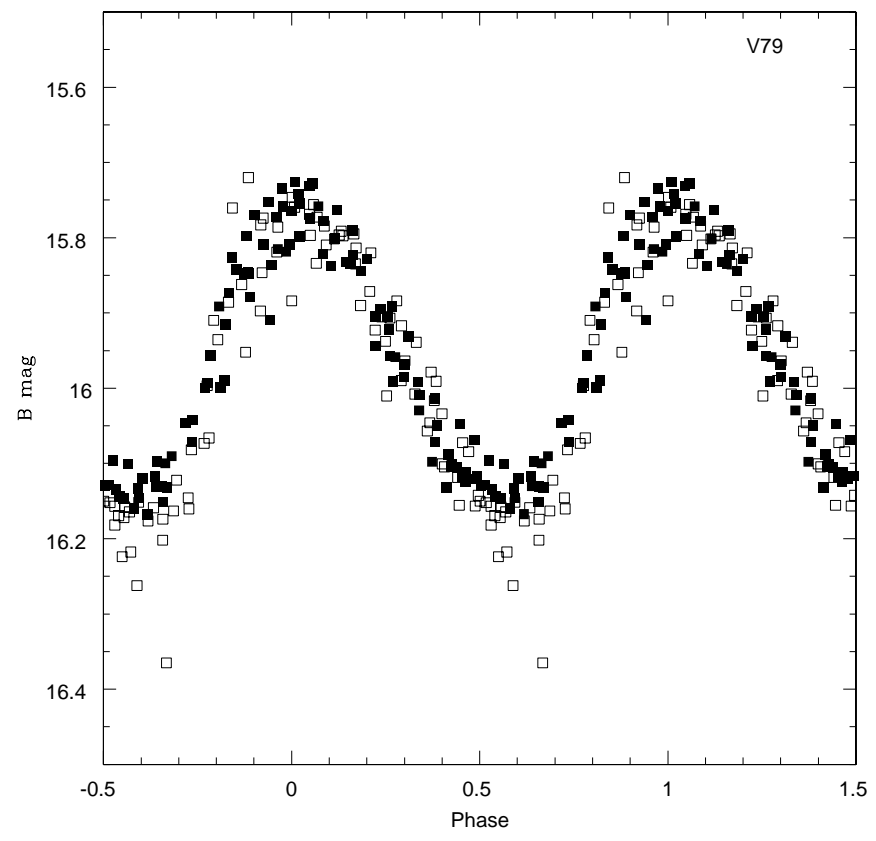

FIG. $8 a$ ing periods in OoII clusters reflects RR Lyrae evolution from the blue to red through the instability strip. This would be consistent with the theoretical predictions of Lee, Demarque, \& Zinn (1990). Smith (1995) warns, however, that although the theory and observations are in general agreement, the data are not adequate to provide confirmation.

Clement et al. (1997) suggest another method for studying horizontal branch evolution: monitoring long-term changes in the pulsation characteristics of double-mode RR Lyrae stars. They present evidence that, for the OoI globular cluster, M3, the first overtones of the RRd stars V68 and

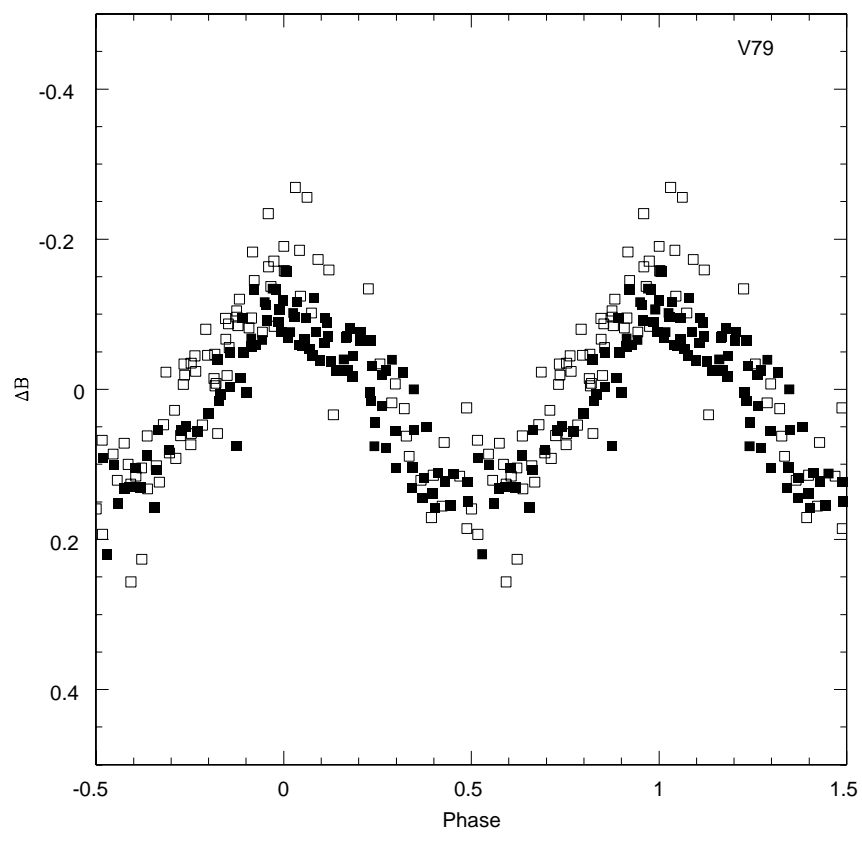

FIG. $8 b$

Fig. 8.- Same as Fig. 7 for V79 


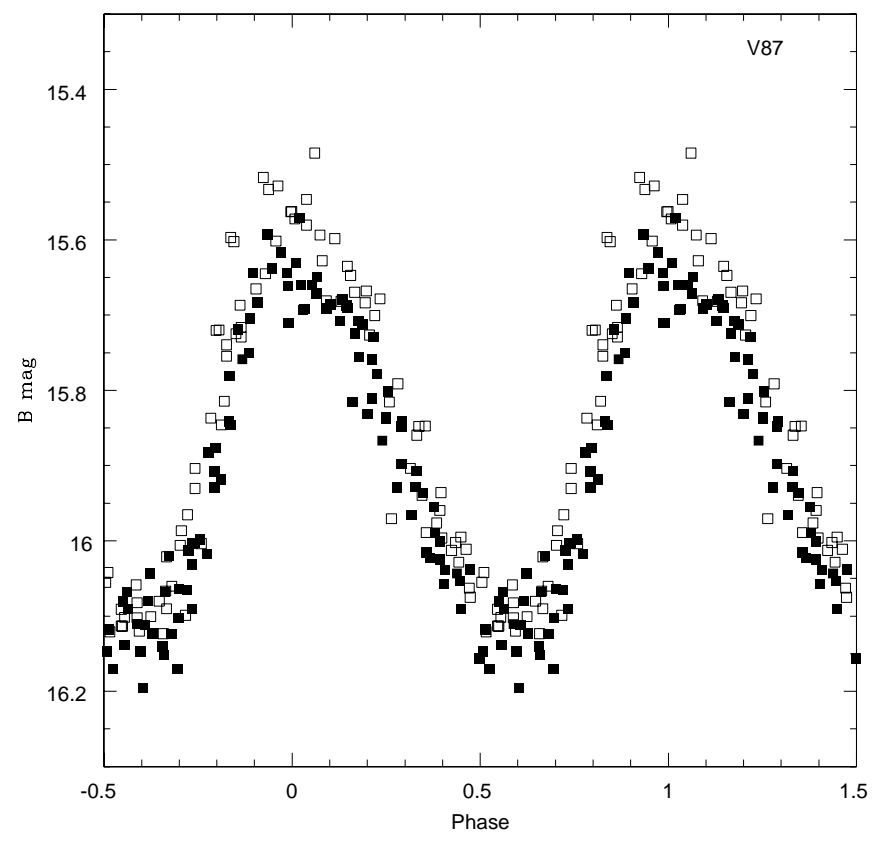

FIG. $9 a$

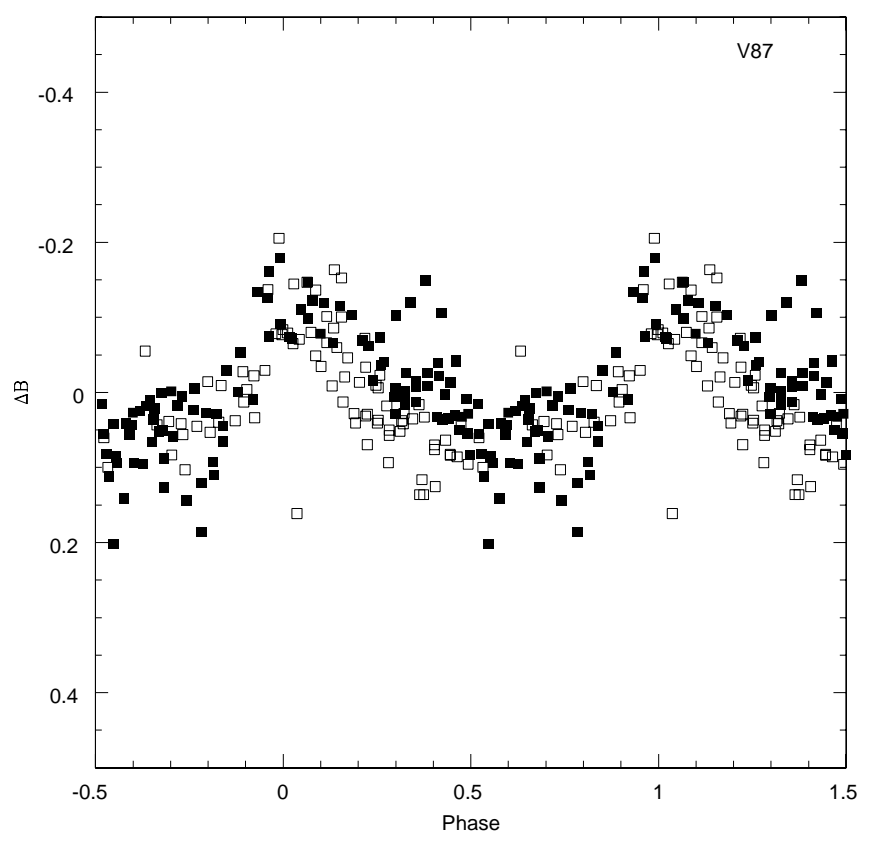

FIG. $9 b$

FIG. 9.- Same as Fig. 7 for V87

V79 are increasing in strength relative to the fundamentalV68 has changed from a dominant fundamental mode to a dominant first overtone and V79 has changed from an $\mathrm{RRab}$ to an RRd variable. This supports the theoretical prediction that the horizontal-branch stars of OoI clusters are evolving blueward. We find that V166 has switched its dominate pulsation mode from fundamental in 1992 to first overtone in 1993. If the mode shift is an evolutionary effect, this would be additional evidence for blueward evolution for M3. Our data also show a possible increase in the first-

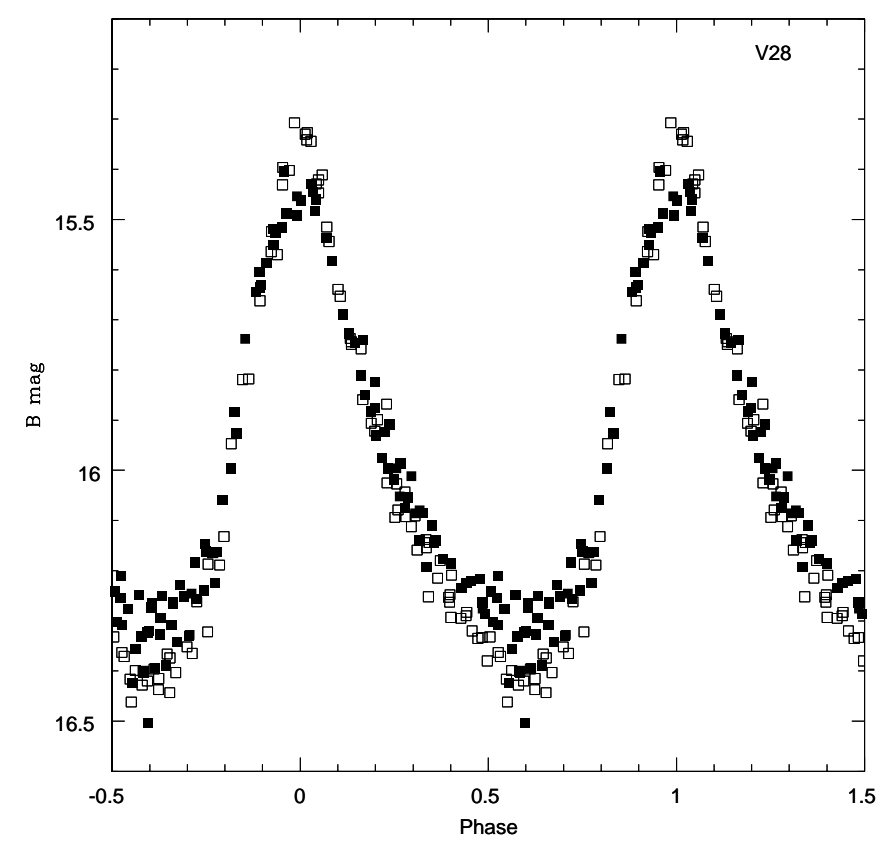

overtone pulsation relative to the fundamental for V99, which again supports blueward evolution.

Most data on RRd variables suggest that they do not show significant changes in their year-to-year behavior and, in fact, tend to be relatively constant in periods and amplitude ratios over decades. Thus, the observation of a switch in dominant pulsation mode in less than $1 \mathrm{yr}$, as our data show for V166, is unexpected. There is, however, some evidence that changes can occur on a short timescale. Clement et al. (1993) reported that V21 in M68 showed RRd behavior during 1986-1988 but not during 1989-1991. Walker

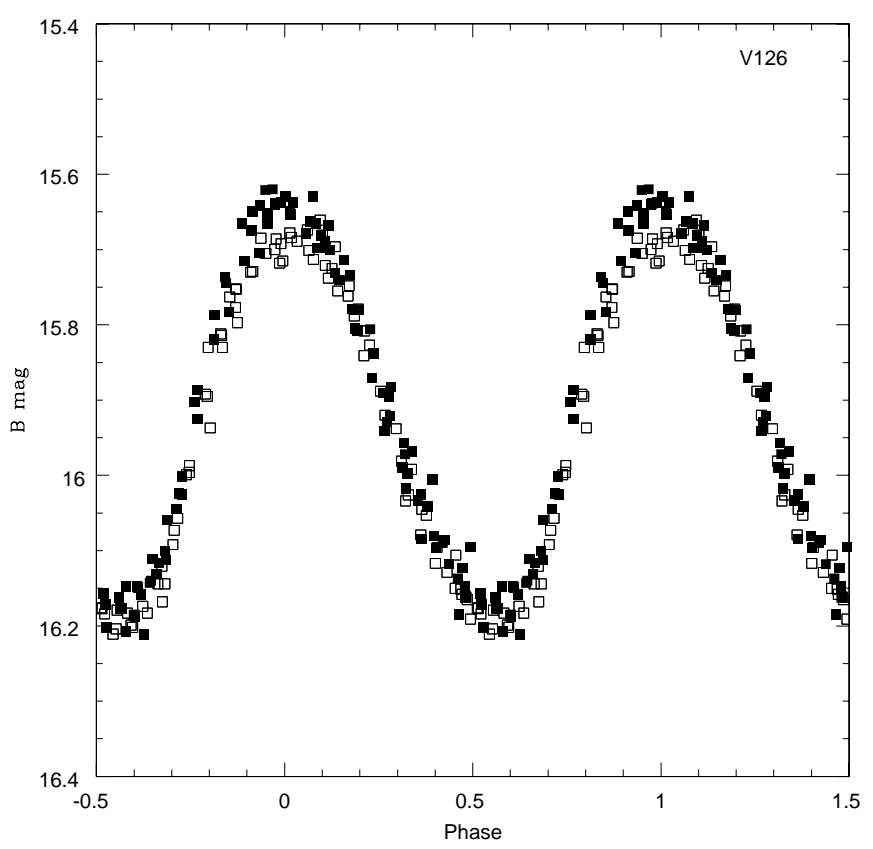

FIG. 10.-Light curves for V28 1992 (open squares) and 1993 (filled squares) data.

FIG. 11.-Same as Fig. 10 for V126 
(1994) found V21 to show double-mode behavior in 1993 data.

The PDM technique assumes that the combined light curve can be described as a simple superposition of the light curves of the fundamental and first-overtone modes. As Purdue et al. (1995) point out for the case of V31 in M15, this may be too simple a model. Our data for the RRd stars in M3 certainly show much more scatter than would be expected with a simple superposition. Clement et al. (1997) point out that prior to 1962 , V79 was classified as a Blazhko variable and offer this as support for the fact that rather than being an effect of magnetic cycles, the Blazhko effect is a "consequence of some type of mixing of pulsational modes" (Smith 1995). The night-to-night variations in the primary and secondary light curves of our data certainly suggest some "Blazhko-like" behavior. Many more data are needed to assess the possible connection between Blazhko behavior and double-mode pulsation. It will be particularly important to locate these two variable types accurately on the color-magnitude and temperaturemagnitude diagrams of the globular clusters in which they appear.

Purdue et al. (1995) have studied the double-mode RR Lyraes in M15 (a prototype OoII cluster, $[\mathrm{Fe} / \mathrm{H}]=-2.15$ ). It is clear that there are some differences in the pulsation properties of the RRd stars in M15 and those in the OoI cluster, M3. They find that while several of the RRd variables in M15 show no significant changes in period or amplitude ratios over the last half-century, the ratio of the first-overtone to fundamental mode of pulsation in V30 and possibly V53 has decreased. They comment that "the increase in the amplitude of the fundamental mode pulsation in V30 may not be a long-term trend indicative of mode switching, but rather a shorter term fluctuation" and that "one might thus envision some instability which over a span of years, can change the double-mode pulsation from a state in which the fundamental mode component is nearly negligible to a state in which the fundamental mode pulsation has an amplitude of about half that of the first overtone mode." For those RRd stars in M3 for which there are clear changes in the amplitude ratio, the result is just the opposite of M15 - it is the strength of the first-overtone pulsation that is increasing relative to the fundamental. This would indicate that the two clusters are evolving in different directions on the horizontal branch, with M3 evolving blueward and M15 redward.

Another difference is in the first-overtone to fundamental amplitude ratios themselves. Purdue et al. conclude that "the concentration of RRd stars with amplitude ratios near two cannot be entirely dismissed as a selection bias." And that "this limitation in the strength of the fundamentalmode oscillation may be of significance to models of double-mode pulsation in RR Lyrae stars." This limitation does not seem to apply to three of the five RRd variables in M3.

It is a pleasure to thank Christine Clement for her helpful discussion. T. M. C. is grateful for support from UNC Charlotte in the form of a semester's Reassignment of Duties grant. Research on RR Lyraes at UNC Chapel Hill is supported by NSF grant AST 96-19831.
Alcock, C., et al. 1997, ApJ, 482, 89

Bailey, S. I. 1913, Harvard Ann., 78

Carretta, E., \& Gratton, R. G. 1997, A\&AS, 121, 95

Clement, C., Ferance, S., \& Simon, N. 1993, ApJ, 412, 183

Clement, C., Hilditch, R. D., Kaluzny, J., \& Rucinski, S. M. 1997, ApJ, 489, 55

Corwin, T. M., Allen, D. M., \& Carney, B. W. 1998, Inf. Bull. Variable Stars, 4548

Cox, A. N., Hudson, S. W., \& Clancy, S. P. 1983, ApJ, 266, 94

Goranskij, V. P. 1981, Inf. Bull. Variable Stars, 2007

Iben, I., Jr., \& Rood, R. T. 1970, ApJ, 161, 587

Landolt, A. U. 1983, AJ, 88, 439

Lee, Y.-W., Demarque, P., \& Zinn, R. 1990, ApJ, 350, 155

\section{REFERENCES}

Martin, W. C. 1942, ApJ, 95, 314

Nemec, J. M. 1985, AJ, 90, 240

Nemec, J. M., \& Clement, C. 1989, AJ, 98, 860

Oosterhoff, P. 1939, Observatory, 62, 104

Purdue, P., Silbermann, N. A., Gay, P., \& Smith, H. A. 1995, AJ, 110, 1712

Sandage, A., Katem, B., \& Sandage, M. 1981, ApJS, 46, 41

Sawyer-Hogg, H. 1973, Publ. David Dunlap Obs., 3, No . 6

Smith, H. A. 1995, RR Lyrae Stars (Cambridge: Cambridge Univ. Press)

Stellingwerf, R. F. 1978, ApJ, 224, 953

Szeidl, B. 1973, Budapest Mitt., No. 63

Walker, A. L. 1994, AJ, 108, 555

Walker, A. L., \& Nemec, J. M. 1996, AJ, 112, 2026 